\section{A TEM Investigation of the Network Structure of Electron Beam Cured Epoxy Polymers}

Richard L. Schalek: Brigitte Defoort," and Lawrence T. Drzal"

Composite Materials and Structures Center and

"Department of Chemical Engineering and Materials Science, Michigan State University

Glassy network epoxies have widespread applications as matrices for advanced composites. As an alternative to thermal curing, electron beam (ebeam) processing has been demonstrated as a very powerful tool for achieving fast and efficient curing $[1,2]$. E-beam curing of epoxy based resins using suitable onium salts as initiators proceeds via a cationic mechanism. The final polymer network properties are highly dependent on processing conditions and curing kinetics. It was demonstrated that under certain processing conditions, heterogeneities can appear in the material, due to the agglomeration of initiator residue. Initially the initiator is miscible with the resin; however, reaction-induced phase separation occurs as the increasing molecular weight of the polymer reduces the solubility of the initiator. The formation of a heterogeneous rather than a homogeneous network depends on the polymerization kinetic, and especially on the vitrification and phase separation rate.

A TEM study was performed to better understand the curing behavior and network formation of e-beam cured epoxies. Tactix 123 resin was mixed with 3 pts/hundred of SarCat CD1012 cationic initiator and e-beam cured using an Acsion Industry 1-10/1 electron linear accelerator (Acsion Industry, Pinawa, Manitoba, Canada). The dose deposited per pass was varied from 5 to $20 \mathrm{kGy}$ for a total dose of $160 \mathrm{kGy}$. The bright field micrograph in Figure 1a shows submicron particles that are associated with holes or embedded in the epoxy matrix. The micrograph in Figure $1 \mathrm{~b}$ shows that the particles are an agglomerate of two morphologies: very small particles and much larger dark particles. Post curing this specimen for 1 hour at $175^{\circ} \mathrm{C}$ results in dissolution of many particles (Figure 2). A particle caught in an intermediate dissolution stage appears in Figure $2 \mathrm{~b}$. XEDS shows these particles have a high concentration of antimony. This suggests that the particles are a byproduct of the initiator. Finally, the TEM micrograph in Figure 3 shows the morphology of the thermally cured epoxy. Though in general the material was featureless, there were some regions populated with submicron particles (A).

\section{References:}

1.) B. Defoort, L. T. Drzal, SAMPE $46^{\text {th }}$ International Symposium, Long Beach, CA. pp2550-2562, (2001).

2.) T. Glauser, M. Johansson, and A. Hult, "Electron-beam Curing of Thick Thermoset Composite Matrices", Polymer, 40, 5297-5302.

\section{Acknowledgements:}

We would like to thank Center for Advanced Microscopy, MSU and the Electron Microbeam Analytical Laboratory, University of Michigan for use of the TEM microscopes. The authors are grateful to $\mathrm{V}$. J. Lopata of Acsion Industries for processing using their electron beam facilities. The electron-beam program conducted at Michigan State University is supported by a research grant from the DOE ORNL RadCure Composite Interphase CRADA program (Cliff Eberle, Program Manager) and, we specifically thank the Department of Energy, Office of Science Laboratory Technology Research Program (Samuel J. Barish, Program Manager.). Oak Ridge National Laboratory is managed by UT-Battelle for the US Department of Energy under contract DE-AC05-00OR22725.

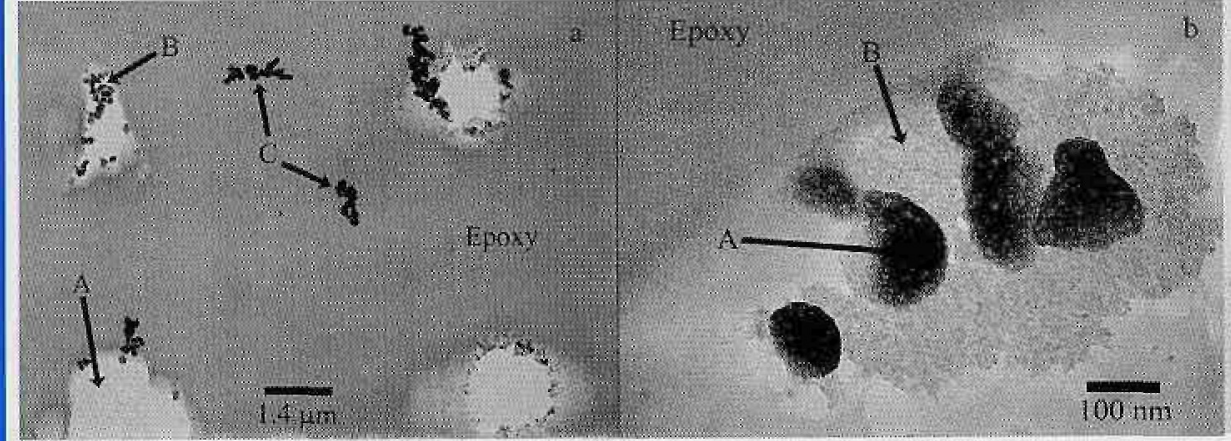

Figure 1a: Bright field TEM micrograph of $160 \mathrm{kGy}$ specimen showing (A) holes, (B) particles near holes, and (C) particles embedded in epoxy.
Figure 1b: Higher magnification of particle in Figure 1a, showing the particle has 2 components $\boldsymbol{A}$ and $\boldsymbol{B}$.

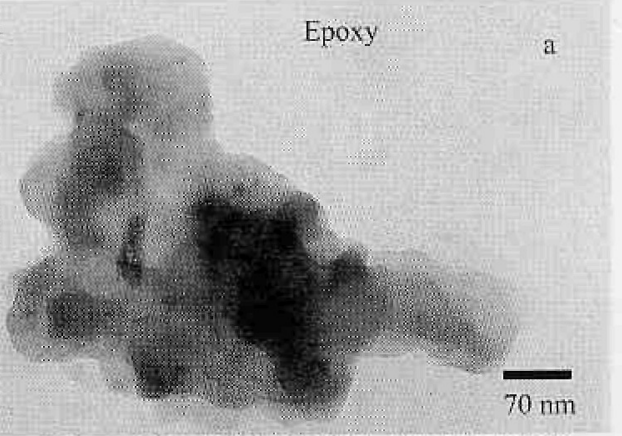

Figure 2a: Bright field TEM micrograph of a particle after e-beam (160 kGy) and post curing for 1 hour at $175^{\circ} \mathrm{C}$.

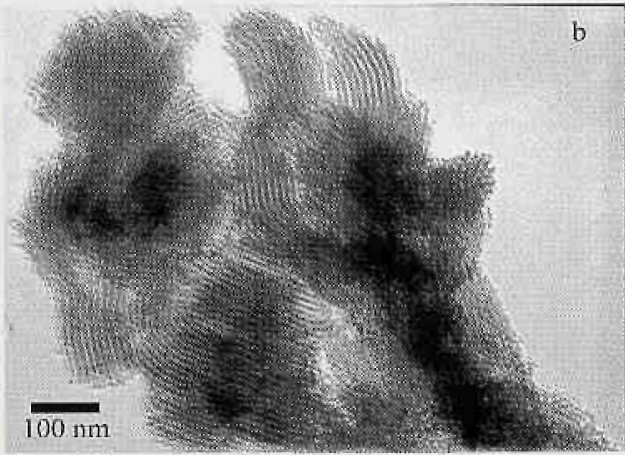

Figure 2b: Bright field TEM micrograph showing the effect of post curing on initially e-beam (160 kGy) cured material.

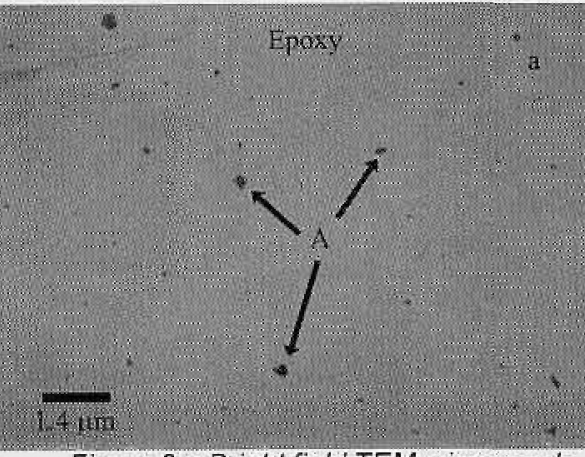

Figure 3a: Bright field TEM micrograph showing the morphology of epoxy thermally cured for 3 hours at $170^{\circ} \mathrm{C}$.

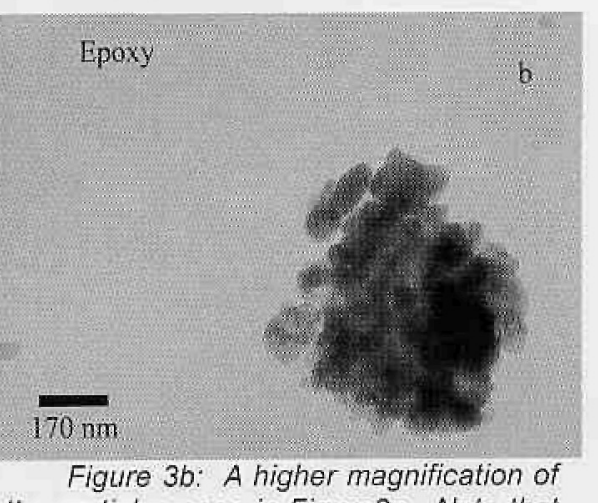

the particles seen in Figure 3a. Note that this is an agglomeration of particles. 


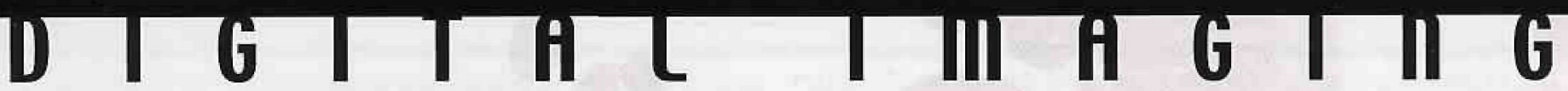

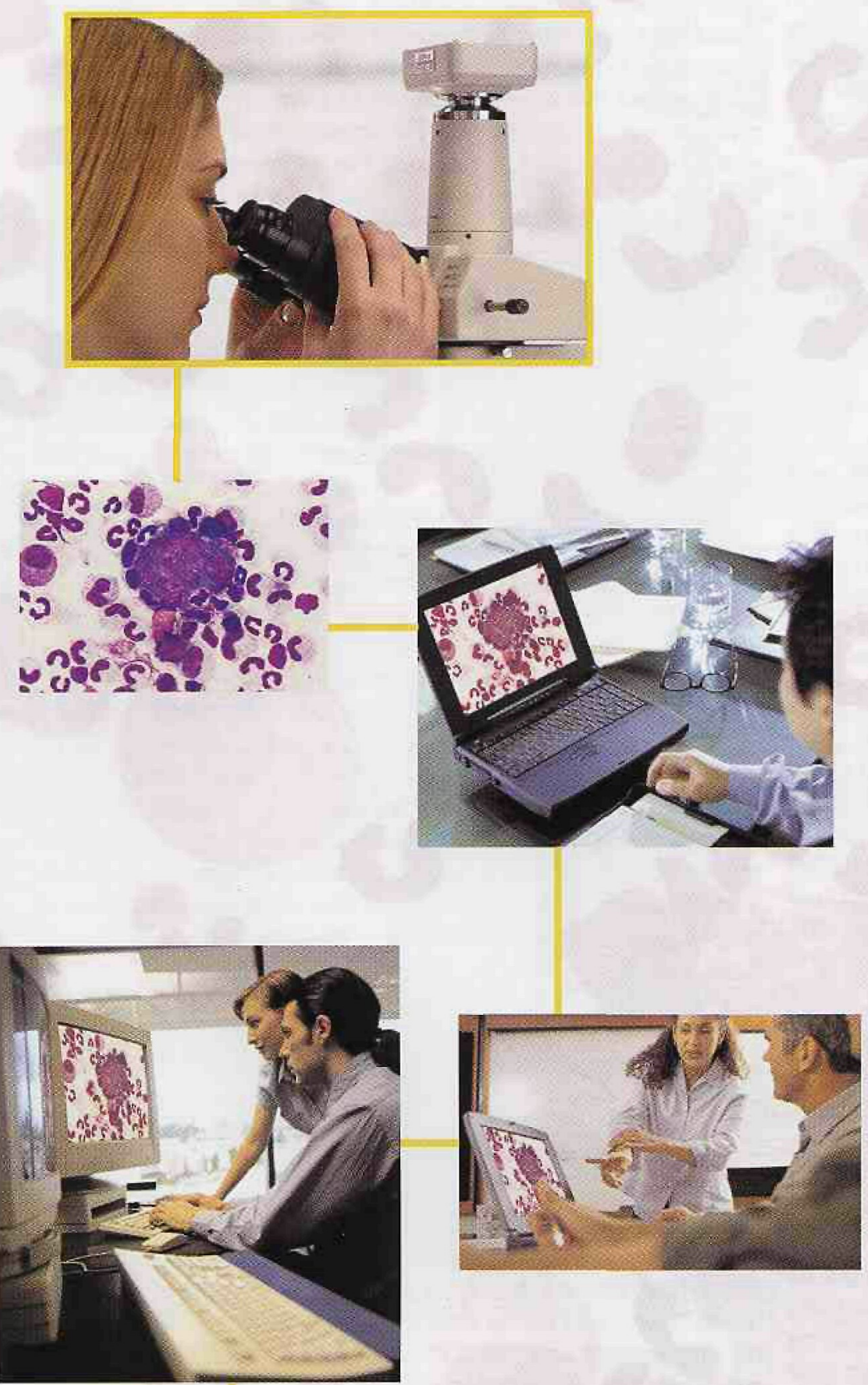

\section{The job's easier when}

everyone sees things the same way.

Now, any image can be shared by everyone anywhere with Nikon's Dn100 network camera.

Here's a terrific way to capture, share and archive your valuable images. Simply plug the Nikon DN 100 Digital Camera directly into a network. Tha's all. It's trouble free, cost effective and there's no need for a PC or special soffware.

The Dn 100 enables simultaneous viewing of images by many people in different locations at megapixel resolution. So, if you need others to see what you see, then this is the perfect digital camera for your microscope.

Features and benefits that make the DN 100 so innovative:

- Users in remote locations can control the camera through a standard Internet browser.

- The camera is a plafform independent network applianceconnects directly to a LAN or WAN.

- Versatile command options include image zoom, auto scaling, draw on screen, and split screen comparison of live and stored images.

To find out more about this highly advanced network camera, call 1-800-52-NIKON, ext. 394 or visit www. nikonusa com

In Canada, call 1-866-99-NIKON.

Visit MicroscopyU at www.nikonusa,com to learn more about digital imaging.
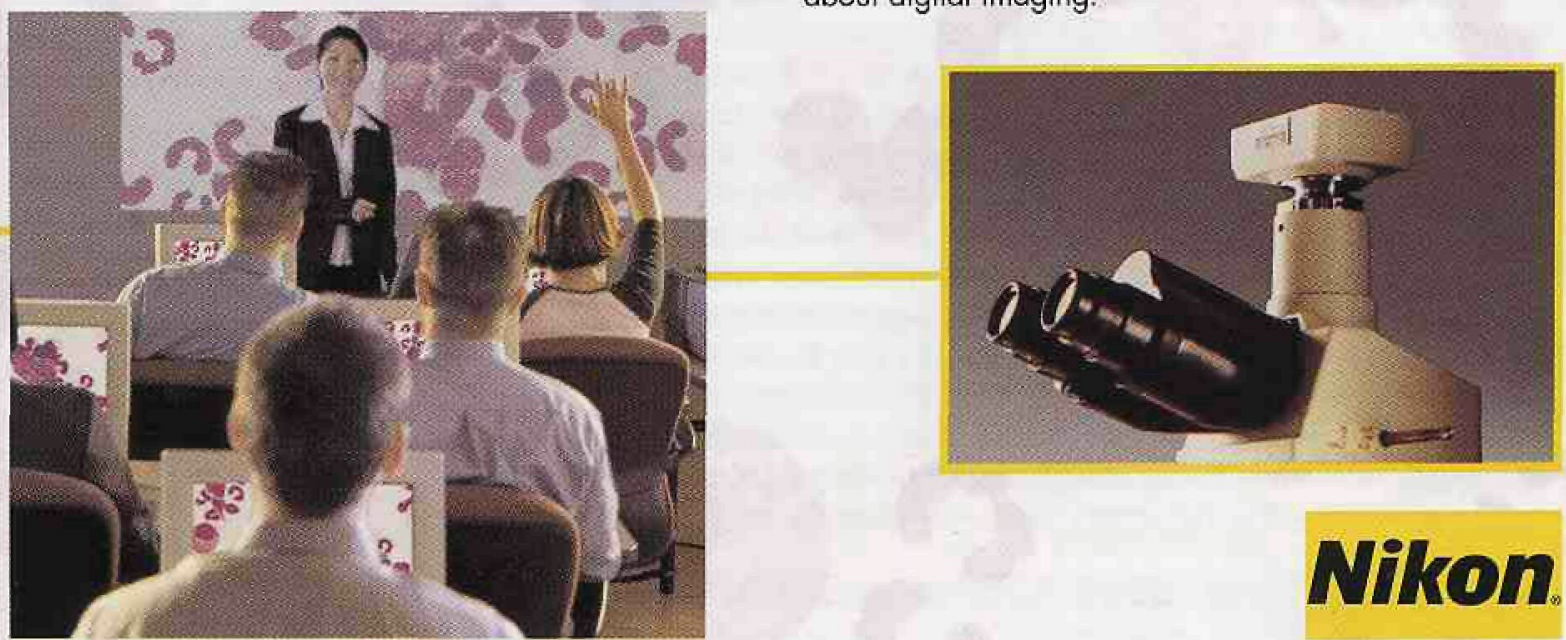